\title{
Rabdomiólise maciça e hepatite aguda secundários ao envenenamento crotálico: um relato de caso
}

\author{
Massive rhabdomyolysis and acute hepatitis secondary to crotalic poisoning: a case report \\ Rabdomiólisis masiva y hepatitis aguda secundaria a la intoxicación por crotálico: informe \\ de un caso
}

Tassio Danilo Rego de Queiroz ${ }^{1 *}$, Natanias Macson da Silva1 ${ }^{1}$, João Lucas Filgueira Nogueira ${ }^{1}$, Ana Carolina Alves de Oliveira ${ }^{1}$, Isaac de Moura Dantas ${ }^{1}$, Liliany Mirelly Bezerra Alves ${ }^{1}$, Thiago Gurgel Regis $^{1}$, Ronaldo Adão da Silva Filho ${ }^{1}$, Hévila Suelen Neri de Lima², Mirna Cavalcante Gurjão ${ }^{3}$.

\section{RESUMO}

Objetivo: Relatar o caso de um paciente que evoluiu com rabdomiólise maciça e hepatite aguda secundários ao acidente crotálico grave. Detalhamento do caso: Paciente do sexo masculino, 27 anos, procedente da zona rural, foi vitimado por picada de cobra durante trabalho no campo. Foi admitido no hospital com quadro clínico bastante comprometido, rebaixamento do nível de consciência, hipotensão e febre. À admissão, os exames laboratoriais bioquímicos e o sumário de urina mostraram comemorativos de rabdomiólise maciça, hepatite aguda e sepse de foco dérmico e o eletrocardiograma mostrou ondas $T$ apiculadas e difusas. Considerações finais: A rabdomiólise constitui um evento clássico do acidente crotálico, todavia, quando maciça, categoriza uma evolução incomum e capaz de induzir à lesão hepática aguda. A hepatotoxicidade é rara e pode estar associada diretamente à peçonha crotálica e, por isso, surgir de maneira isolada; ou ser agravada pela rabdomiólise. Assim, novos exames complementares com foco na avaliação da integridade hepática devem ser inseridos na condução diagnóstica. Após o reconhecimento da hepatite aguda, a preservação da função hepática deve ser um dos objetivos no transcurso terapêtico, a fim de garantir um melhor prognóstico e redução da taxa de mortalidade.

Palavras-chave: Acidente ofídico, Crotalus, Rabdomiólise, Lesão hepática por substâncias.

\begin{abstract}
Objective: To report the case of a patient who developed massive rhabdomyolysis and acute hepatitis secondary to a severe crotalic accident. Case details: A 27-year-old male patient from a rural area was victimized by a snake bite while working in the field. He was admitted to the hospital with a severely compromised clinical condition, decreased level of consciousness, hypotension and fever. On admission, biochemical laboratory tests and urine summary showed massive rhabdomyolysis, acute hepatitis, and sepsis of dermal focus, and the electrocardiogram showed apiculate and diffuse $T$ waves. Final considerations: Rhabdomyolysis is a classic event of the crocal accident, however, when massive, it categorizes an unusual evolution capable of inducing acute liver injury. Hepatotoxicity is rare and can be directly associated with the venomous venom and therefore appear in isolation; or be aggravated by rhabdomyolysis. Thus, new complementary tests focusing on the evaluation of liver integrity should be inserted in the diagnostic management. After the recognition of acute hepatitis, the preservation of liver function should be one of the goals in the course of therapy to ensure a better prognosis and reduce mortality rate.
\end{abstract}

Keywords: Ophidian accident, Crotalus, Rhabdomyolysis, Hepatic substance injury.

\section{RESUMEN}

Objetivo: Comunicar el caso de un paciente que desarrolló rabdomiólisis masiva y hepatitis aguda secundaria a un accidente crotálico grave. Detalles del caso: Un paciente varón de 27 años de una zona rural fue víctima de una mordedura de serpiente mientras trabajaba en el campo. Ingresó en el hospital con un estado clínico muy comprometido, disminución del nivel de conciencia, hipotensión y fiebre. Al ingreso, los exámenes bioquímicos de laboratorio y el resumen de orina mostraron rabdomiólisis masiva, hepatitis aguda y sepsis de foco dérmico y el electrocardiograma mostró ondas $T$ apiculadas y difusas.

\footnotetext{
${ }^{1}$ Universidade do Estado do Rio Grande do Norte (UERN), Mossoró - RN.

*E-mail: tassioqueiroz@alu.uern.br

2 Universidade Federal Rural do Semi-Árido (UFERSA), Mossoró - RN.

3 Hospital Wilson Rosado, Mossoró - RN.
} 
Consideraciones finales: La rabdomiólisis es un evento clásico del accidente crocal, sin embargo, cuando es masiva, categoriza una evolución inusual capaz de inducir una lesión hepática aguda. La hepatotoxicidad es poco frecuente y puede estar directamente asociada al veneno y, por lo tanto, aparecer de forma aislada; o verse agravada por la rabdomiólisis. Por lo tanto, los nuevos exámenes complementarios centrados en la evaluación de la integridad hepática deben incluirse en el manejo diagnóstico. Tras el reconocimiento de la hepatitis aguda, la preservación de la función hepática debe ser uno de los objetivos en el curso de la terapia para asegurar un mejor pronóstico y reducir la tasa de mortalidad.

Palabras clave: Accidente ofídico, Crotalus, Rabdomiólisis, Lesión de la Sustancia Hepátic.

\section{INTRODUÇÃO}

Dentre os principais gêneros envolvidos nos acidentes ofídicos no Brasil, o gênero Crotalus sp corresponde a $86,8 \%$ dos casos brasileiros e das possibilidades de geração de diferentes apresentações clínicas, além de ser responsável por 8,9\% das ocorrências e apresentar a maior mortalidade, que é quatro vezes maior que a do gênero Bethrops sp. (MATOS RR e IGNOTTI E, 2020).

A farmacologia geral e a composição do veneno das várias espécies de Crotalus no Brasil são muito semelhantes, mas existem algumas variabilidades intraespecíficas e interespecíficas. Os venenos são essencialmente compostos por quatro toxinas: crotoxina (ação neurotóxica e miotóxica), giroxina e convulxina (ação coagulante) e crotamina (ação miotóxica) (FRARE BT, et al., 2019).

Nas primeiras horas de evolução clínica, as manifestações neurológicas caracterizam-se por ptose palpebral (uni ou bilateral), flacidez muscular facial, diâmetro pupilar alterado, oftalmoplegia, visão turva e/ou diplopia (MENDONÇA-DA-SILVA I, et al., 2017; PUCCA MB, et al., 2021). Ademais, o quadro também pode apresentar coagulação alterada em 55,5\% dos pacientes (SOUZA LA, et al., 2021).

Por outro lado, a ação miotóxica produz lesões na musculatura esquelética com liberação de enzimas, eletrólitos e mioglobina na corrente sanguínea (rabdomiólise), o que pode causar mialgia, mioglobinúria e colúria (LIMA SC, et al., 2018). Inclusive, essa mionecrose sistêmica contribui para a Lesão Renal Aguda (LRA), a qual é a principal complicação do acidente crotálico e pode surgir até 48 horas após a inoculação do veneno (CARVALHO LH, et al., 2019).

Sabe-se que as complicações sistêmicas, como o comprometimento renal, podem contribuir para o pior prognóstico (OLIVEIRA IS, et al., 2019). Nesse cenário, embora seja uma evolução pouco descrita na literatura, estudos sugerem que o veneno crotálico apresenta algum componente hepatotóxico ainda não identificado, mas possivelmente relacionado com desequilíbrios da homeostase redox, além de sobrecarga hepática frente às necessidades fisiológicas e patológicas, mediante rabdomiólise associada (FRARE BT, et al., 2019).

De acordo com as manifestações clínicas evidentes, o quadro de envenenamento crotálico pode ser classificado em leve, moderado e grave (VAZ VHS, et al., 2020). Nos casos classificados como leves, há manifestações neuroparalíticas discretas, ausência de mialgia e de comprometimento do trato urinário. Por outro lado, os casos moderados cursam com sinais neuroparalíticos evidentes, mialgia e mioglobinúria discreta. Por fim, as ocorrências mais graves apresentam mialgia intensa, sintomas neuroparalíticos críticos, mioglobinúria maciça e oligúria (FARIA G e LIMA AAM, 2019).

A terapêutica específica para o acidente crotálico é a administração de Soro Anticrotálico (SAC), via endovenosa, na quantidade de 5, 10 e 20 ampolas para os casos leves, moderados e graves, respectivamente; na falta deste, poderá ser empregado o Soro Antibotrópico-Crotálico (SABC) (FUNASA, 2001, apud FRARE BT, et al., 2019).

Embora as ações da peçonha crotálica sejam conhecidas, alguns casos de envenenamento crotálico podem apresentar acometimentos atípicos que precisam ser elucidados, como a associação entre mionecrose e lesão hepática; o que pode modificar o arsenal diagnóstico-terapêutico, sobretudo nos quadros graves (FRARE BT, et al., 2019). Diante disso, este estudo de caso buscou descrever e analisar, à luz da literatura, um caso de acidente crotálico com rabdomiólise maciça e hepatite aguda. 


\section{DETALHAMENTO DO CASO}

Este estudo foi aprovado pelo Comitê de Ética e Pesquisa da Universidade do Estado do Rio Grande do Norte, sob o parecer de número 4.155.143 e CAAE: 34035720.6.0000.5294, no dia 14 de julho de 2020.

Paciente do sexo masculino, 27 anos, procedente da zona rural do Alto Oeste Potiguar, relata que foi vitimado por picada de cobra, aparentemente uma cascavel devido ao chocalho descrito pelo paciente, na tarde do dia 05/06/2020, durante trabalho no campo. Mesmo diante do evento ofídico, não buscou cuidados médicos e somente na manhã do dia seguinte procurou uma unidade de saúde em seu município, sendo transferido imediatamente para o hospital de referência da região.

Assim, deu entrada na unidade às 08h30min do dia 06/05/2020 com quadro clínico bastante comprometido, apresentando rebaixamento do nível de consciência, hipotensão (90x50 mmHg) e febre. Foram identificadas lesões puntiformes discretas na pele, localizadas na região dorsolateral do pé direito (sem necrose evidente) e sinais sugestivos de presas de serpente. Dessa forma, por aparentar uma cascavel pelo relato do vitimado, o paciente, inicialmente, foi tratado com reposição volêmica vigorosa com soro fisiológico $0,9 \%$ e administradas 4 ampolas de soro antibotrópico-antilaquético, bem como 4 ampolas de soro anticrotálico, contemplando de forma geral os possíveis tipos de serpentes da região.

Os exames laboratoriais e sumário de urina mostraram comemorativos de rabdomiólise maciça, hepatite aguda e sepse de foco dérmico (Tabela 1 e Tabela 2). O Eletrocardiograma (ECG) mostrou ondas $T$ apiculadas e difusas.

Tabela 1 - Painel laboratorial de exames bioquímicos e hematológicos de um paciente vítima de acidente crotálico.

\begin{tabular}{|c|c|c|c|c|}
\hline Séries & Exames & $\begin{array}{l}\text { SAU }-06 / 05 / 20 \\
\text { às } 08: 40 \text { horas }\end{array}$ & $\begin{array}{l}\text { UTI - 06/05/20 } \\
\text { às 17:01 horas }\end{array}$ & Valor de referência \\
\hline \multirow{6}{*}{ Eritrograma } & Hemácias $\left(10^{6} / \mathrm{mm}^{3}\right)$ & 6,0 milhões $/ \mathrm{mm}^{3}$ & 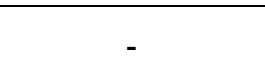 & $\begin{array}{c}4,5-6,1 \\
\text { milhões } / \mathrm{mm}^{3}\end{array}$ \\
\hline & Hemoglobina (g/dl) & $20,9 \mathrm{~g} / \mathrm{dL}$ & - & $13-18 \mathrm{~g} / \mathrm{dL}$ \\
\hline & Hematócrito $(\%)$ & $60,9 \%$ & - & $40-54 \%$ \\
\hline & VCM (fl) & $101,2 \mathrm{fL}$ & - & $80-96 \mathrm{fL}$ \\
\hline & HCM (pg) & $34,7 \mathrm{pg}$ & - & $27,5-33,2 p g$ \\
\hline & $\mathrm{CHCM}(\mathrm{g} / \mathrm{dl})$ & $34,3 \mathrm{~g} / \mathrm{dL}$ & - & $31-35 \mathrm{~g} / \mathrm{dL}$ \\
\hline \multirow{7}{*}{ Leucograma } & $\begin{array}{l}\text { Leucócitos totais / } \\
\mathrm{mm}^{3}\end{array}$ & 32.700 & - & $4.000-11.000$ \\
\hline & $\begin{array}{l}\text { Neutrófilos } \\
\text { bastonetes (\%) }\end{array}$ & 30 & - & $1-4$ \\
\hline & $\begin{array}{l}\text { Neutrófilos } \\
\text { seamentados (\%) }\end{array}$ & 64 & - & $40-65$ \\
\hline & Eosinófilos (\%) & 0 & - & $1-5$ \\
\hline & Basófilos (\%) & 0 & - & $0-1$ \\
\hline & Linfócitos (\%) & 4 & - & $22-45$ \\
\hline & Monócitos (\%) & 2 & - & $2-10$ \\
\hline \multirow{4}{*}{ Hemostasia } & Plaquetas $\left(/ \mathrm{mm}^{3}\right)$ & $247.000 / \mathrm{mm}^{3}$ & - & $150.000-450.000$ \\
\hline & Tempo de & $/, 5 / \mathrm{TL}$ & & 6,L- II,8/IL \\
\hline & sangramento & $1 \mathrm{~min} 0 \mathrm{seg}$ & - & 1 a 3 min \\
\hline & $\begin{array}{l}\text { Tempo de } \\
\text { coagulação }\end{array}$ & $\begin{array}{l}\text { Incoagulável (não } \\
\text { coagulou até } 30 \\
\text { minutos) }\end{array}$ & $\begin{array}{c}\text { Incoagulável } \\
\text { (não coagulou } \\
\text { até } 30 \text { minutos) }\end{array}$ & 5 a $10 \min$ \\
\hline \multirow{7}{*}{$\begin{array}{l}\text { Exames } \\
\text { bioquímicos }\end{array}$} & Amilase U/L & $52 \mathrm{U} / \mathrm{L}$ & - & 30 a $110 \mathrm{U} / \mathrm{L}$ \\
\hline & CPK U/L & 89. $320 \mathrm{U} / \mathrm{L}$ & - & 23 a $300 \mathrm{U} / \mathrm{L}$ \\
\hline & LDH U/L & $10.345,0 \mathrm{U} / \mathrm{L}$ & - & $313 \mathrm{U} / \mathrm{L}$ a $618 \mathrm{U} / \mathrm{L}$ \\
\hline & Ureia mg / dL & 34 mg / dL & - & స 19,3 a $42,9 \mathrm{mg} / \mathrm{dL}$ \\
\hline & Creatinina mg / dL & $1,2 \mathrm{mg} / \mathrm{dL}$ & - & 0,66 a $1,25 \mathrm{mg} / \mathrm{dL}$ \\
\hline & TGP/ALT U/L & $1.969 \mathrm{U} / \mathrm{L}$ & - & $0^{\lambda}<38 \mathrm{U} / \mathrm{L}$ \\
\hline & TGO/AST U/L & $6.904 \mathrm{U} / \mathrm{L}$ & - & t até $38 \mathrm{U} / \mathrm{L}$ \\
\hline
\end{tabular}

Legenda: SAU: Serviço de Atendimento de Urgência; UTI: Unidade de Terapia Intensiva.

Fonte: Queiroz TDR, et al., 2021. 
Tabela 2 - Sumário de Urina I de um paciente vítima de acidente crotálico.

\begin{tabular}{|c|c|c|c|}
\hline Séries & Exames & $\begin{array}{c}\text { SAU }-06 / 05 / 20 \text { às } \\
08: 40 \text { horas }\end{array}$ & Valor de referência \\
\hline Exame físico & $\begin{array}{l}\text { Cor } \\
\text { Aspecto } \\
\text { pH } \\
\text { Reação } \\
\text { Densidade } \\
\text { Odor }\end{array}$ & $\begin{array}{c}\text { Amarelo Claro } \\
\text { Turvo } \\
6.5 \\
\text { Acida } \\
1.015 \\
\text { Sui generis }\end{array}$ & $\begin{array}{c}\text { Límpido } \\
5.0 \text { a } 7.0 \\
\text { Acida } \\
\text { de } 1.005 \text { a } 1035 \\
-\end{array}$ \\
\hline Exame químico & $\begin{array}{l}\text { Hemoglobina } \\
\text { Nitrito } \\
\text { Bilirrubina } \\
\text { Glicose } \\
\text { Corpos Cetônicos } \\
\text { Urobilinogênio } \\
\text { Proteínas }\end{array}$ & $\begin{array}{c}\text { Positivo (+++) } \\
\text { Negativo } \\
\text { Negativo } \\
\text { Negativo } \\
\text { Negativo } \\
\text { Negativo } \\
\text { Positivo }(++)\end{array}$ & $\begin{array}{c}\text { Negativo } \\
\text { Negativo } \\
\text { Negativo } \\
\text { Negativo } \\
\text { Negativo } \\
\text { Normal } \\
\text { Negativo }\end{array}$ \\
\hline $\begin{array}{l}\text { Exame microscópico do } \\
\text { sedimento }\end{array}$ & $\begin{array}{l}\text { Hemácias } \\
\text { Leucócitos (piócitos) } \\
\text { Células epiteliais } \\
\text { Filamentos de Muco } \\
\text { Flora bacteriana } \\
\text { Cristais } \\
\text { Cilindros }\end{array}$ & $\begin{array}{c}18 \text { a 20/campo } \\
1 \text { a } 2 / \text { campo } \\
\text { Raras } \\
\text { Ausente } \\
\text { Raras } \\
\text { Vários uratos amorfos } \\
\text { Vários granulosos }\end{array}$ & $\begin{array}{l}\text { Até } 3 \text { por campo } \\
\text { Até } 5 \text { por campo } \\
\text { Ausentes } \\
\text { Não se aplica } \\
\text { Ausentes } \\
\text { Ausentes } \\
\text { Ausentes }\end{array}$ \\
\hline
\end{tabular}

Legendas: SAU: Serviço de Atendimento de Urgência. Fonte: Queiroz TDR, et al., 2021.

O paciente foi admitido na Unidade de Terapia Intensiva (UTI) às $16 \mathrm{~h}(06 / 05 / 2020)$, com estado geral grave, comatoso, taquicárdico, taquipneico e saturando a $98 \%$ (oximetria de pulso); foi ofertado de oxigênio por cateter nasal. Ao exame físico geral, o paciente estava desorientado, desidratado $(2+/ 4+)$, normocorado, acianótico e afebril. Ao exame neurológico, pupilas isocóricas e fotorreagentes, a Escala de Coma de Glasgow (ECG) não registrada.

Ao exame respiratório, tórax simétrico com expansibilidade prejudicada, sem ruídos adventícios e frequência respiratória de 22 incursões por minuto. Ao exame cardiovascular, ritmo cardíaco regular em dois tempos, bulhas normofonéticas sem sopros; pulso cheio, regular e simétrico, perfusão periférica preservada, enchimento capilar < 2 segundos, Pressão Arterial (PA) de $146 \times 115 \mathrm{mmHg}$, Frequência Cardíaca (FC) de 122 Batimentos Por Minuto (bpm). Ao exame abdominal, abdome plano, flácido, indolor à palpação e ruídos hidroaéreos presentes. Na avaliação da diurese, evoluiu com hematúria (Sonda Vesical de Demora). Após tal avaliação, iniciou-se antibioticoterapia empírica com Ceftriaxona e Ciprofloxacino.

Às 20hs, o paciente evoluiu com rebaixamento súbito do nível de consciência, parada respiratória e, em seguida, cardiorrespiratória em assistolia. O paciente foi reanimado após 10 minutos de manobras de ressuscitação. Com estado geral gravíssimo, apresentou PA de 111 X 66, FC de 122 bpm, temperatura de $37,6^{\circ} \mathrm{C}$ e acidose metabólica (Tabela 3). Cerca de $1 \mathrm{~h}$ e $30 \mathrm{~min}$ após a primeira PCR, apresentou um segundo episódio, com duração de 14 minutos, e posteriormente com retorno à circulação espontânea mediante uso de drogas vasoativas.

Tabela 3 - Gasometria Arterial de um paciente vítima de acidente crotálico.

\begin{tabular}{lcc}
\hline Parâmetros & UTI - 06/05/20 às 20:15 horas & Valor de referência \\
\hline Ph & 6,867 & $7,35-7,45$ \\
pCO2 & $113,9 \mathrm{mmHg}$ & $35-45 \mathrm{mmHg}$ \\
pO2 & $69,1 \mathrm{mmHg}$ & $83-108 \mathrm{mmHg}$ \\
$\mathrm{HCO} 3$ & $20,9 \mathrm{mmHg}$ & $22-28 \mathrm{mmHg}$ \\
$\mathrm{SO} \%$ & 73,3 & $>92 \%$ \\
Änion Gap (mEq/L) & $15 \mathrm{mEq} / \mathrm{L}$ & $6-16 \mathrm{mEg} / \mathrm{L}$ \\
$\mathrm{Hematócrito} \mathrm{( \% )}$ & $42 \%$ & $35-49 \%$ \\
$\mathrm{Na}+(\mathrm{mmol} / \mathrm{L})$ & $133,1 \mathrm{mmol} / \mathrm{L}$ & $135-145 \mathrm{mmol} / \mathrm{L}$ \\
$\mathrm{K}^{+}(\mathrm{mmol} / \mathrm{L})$ & $6,20 \mathrm{mmol} / \mathrm{L}$ & $3,5-5,10 \mathrm{mmol} / \mathrm{L}$ \\
$\mathrm{Cl}(\mathrm{mmol} / \mathrm{L})$ & $103,5 \mathrm{mmol} / \mathrm{L}$ & $98-106 \mathrm{mmol} / \mathrm{L}$ \\
$\mathrm{ICa}(\mathrm{mmol} / \mathrm{L})$ & $0,76 \mathrm{mmol} / \mathrm{L}$ & $1,09-1,30 \mathrm{mmol} / \mathrm{L}$ \\
Glicose $(\mathrm{mg} / \mathrm{dL})$ & $274 \mathrm{mg} / \mathrm{dL}$ & $65-99 \mathrm{mg} / \mathrm{dL}$ \\
Lactato $(\mathrm{mmol} / \mathrm{L})$ & $9,2 \mathrm{mmol} / \mathrm{L}$ & $0,7-2,5 \mathrm{mmol} / \mathrm{L}$ \\
\hline
\end{tabular}

Legendas: UTI: Unidade de Terapia Intensiva. Fonte: Queiroz TDR, et al., 2021. 
Após os dois episódios, teve piora importante do quadro clínico, com perfusão periférica comprometida, palidez cutânea e extremidades frias. Pupilas médio-fixas em midríase e não reagentes aos estímulos luminosos. Às 02h e 40min apresentou mais um episódio de PCR e, em seguida, óbito.

\section{DISCUSSÃO}

O acidente ofídico possui importância em nível de saúde pública, sobretudo, nas zonas rurais dos países tropicais. Em 2017, a OMS inseriu a mordedura por serpentes venenosas no rol de doenças tropicais negligenciadas, o que solicita, por parte dos gestores, medidas e políticas públicas enérgicas (GUTIÉRREZ JM, et al., 2017).

Os acidentes com serpentes ocorrem em todo o território brasileiro e adquire maior notabilidade na saúde nacional ao não ser implantada a conduta precoce e oportuna pela soroterapia. Foram contabilizados, entre 2000 e 2016, 443.912 eventos ofídicos no Brasil, dentre os quais evoluíram a óbito 1815 acidentes, com maior destaque numérico para a região norte (MINISTÉRIO DA SAÚDE, 2021).

O Ministério da Saúde contabilizou 31.395 acidentes ofídicos em 2020 em suas plataformas de notificação. Em território nacional, as jararacas contemplam $70 \%$ dos eventos; as cascavéis, em torno de $9 \%$; as surucucus $1,5 \%$; e as corais verdadeiras registram menos de $1 \%$ da totalidade, merecendo destaque as populações mais suscetíveis aos acidentes ofídicos, notadamente os indígenas e os profissionais que atuam no campo (MINISTÉRIO DA SAÚDE, 2021).

Em 2014, o coeficiente de letalidade no acidente crotálico atingiu mais de 1\%, possuindo, assim, a maior taxa de mortalidade notificada, o que varia em conformidade com a rapidez assistencial a administração da soroterapia (MINISTÉRIO DA SAÚDE, 2014). Nessa perspectiva de complicações e maior óbito pela peçonha crotálica, destacam-se, para além da Lesão Renal Aguda (LRA), a rabdomiólise, a qual pode se estabelecer em diferentes níveis de gravidade, e os acometimentos de outros sítios corporais (ROSA AC, et al., 2021).

A rabdomiólise é definida como uma lesão muscular que resulta na liberação de conteúdos celulares potencialmente tóxicos na circulação sanguínea (HEARD H e BARKER J, 2016). Tais conteúdos incluem metabólitos como potássio, fosfato e urato, enzimas como Creatine PhosphoKinase (CPK) e Lactate DeHydrogenase (LDH) e proteínas intracelulares como mioglobina. Em casos de maior gravidade, a rabdomiólise pode desencadear síndrome compartimental, coagulação intravascular disseminada, LRA e distúrbio eletrolítico; neste último caso, o ECG foi útil no seguimento do paciente, pois revelou ondas $T$ apiculadas e difusas, o que sugere quadro de hipercalemia. Sabe-se que esse distúrbio pode causar arritmias malignas e instabilidade hemodinâmica (PASTORE NETO M, et al., 2018).

A rabdomiólise no acidente crotálico é demonstrada por meio do laboratório enzimático centrado em três enzimas: Aspartato Amino Transferase (AST), LDH e CPK, sendo esta última o marcador mais sensível (TORRES PA, et al., 2015). Em razão disso, o diagnóstico é realizado pela mensuração da CPK sérica encontrada em uma concentração cinco vezes maior que o limite superior do valor de referência, equivalente a valores acima $1000 \mathrm{U} / \mathrm{L}$ (HEARD H e BARKER J, 2016).

À luz da literatura, o aumento da CPK ocorre de maneira precoce, com elevação máxima de pico nas primeiras 24 horas após o acidente. Por outro lado, o aumento da LDH é lento e gradual e, portanto, constitui um exame para o diagnóstico tardio de envenenamento crotálico (SOTO-BLANCO B e MELO MM, 2018). De acordo com os exames admissionais desse estudo de caso, ambas as enzimas se encontraram elevadas simultaneamente, o que pode ser explicado pela procura de atendimento médico tardio e/ou peculiaridades do veneno, incluindo concentração dos seus componentes e o acometimento de diferentes sítios corpóreos produtores da LDH, como o tecido muscular (rabdomiólise maciça), renal e hepático.

Apesar de a LDH ser um marcador inespecífico de lesão hepática, os dados sugerem que o seu aumento repentino, e em altos níveis séricos, pode indicar um comprometimento para além do músculo esquelético, como uma possível lesão hepática aguda (LIM AKH, 2020). Para sustentar tal hipótese, outros marcadores de lesão hepática foram avaliados e encontram-se elevados (Tabela 1). 
Um estudo brasileiro recente analisou os efeitos locais e sistêmicos dos venenos de espécies do gênero Crotalus sp, popularmente conhecidas como cobras cascavéis, constatando o aumento dos níveis de CKTOTAL, CK-MB, LDH e AST, sustentando o quadro de rabdomiólise. Contudo, os mesmos autores observaram que as enzimas Alanina Amino Transferase (ALT), Gama Glutamil Transferase (GGT) e Fosfatase Alcalina (FA) apresentaram-se elevadas e justificaram a presença de comprometimento hepático associado ao quadro (CARVALHO LH, et al., 2019).

Em outra pesquisa, Barraviera B, et al. (1989) identificaram, em um exame anatomopatológico, comemorativos de necrose hepática extensa, o que corrobora com os achados de França RF, et al. (2009) após observarem altos níveis séricos de ALT, AST, LA e GGT associados a um quadro de hepatite aguda.

Sabe-se que as aminotransferases são biomarcadores úteis para o reconhecimento de lesão hepática. A AST pode ser encontrada tanto no meio citoplasmático quanto no interior das mitocôndrias de hepatócitos, porém é encontrada em outros sítios como musculatura cardíaca, musculatura esquelética e rins e possui menor sensibilidade e especificidade para o fígado (LIM AKH, 2020).

Por outro lado, a ALT é uma enzima citosólica mais hepatoespecífica em decorrência da alta concentração no parênquima hepático, sendo encontrada no músculo esquelético, porém em concentrações muito baixas (JO KM, et al., 2019; LIM AKH, 2020; MORILES KE e AZER SA, 2020). Inclusive, a dosagem da ALT é mais específica para revelar dano/inflamação hepática quando comparada com a relação TGO/AST, constituindo-se como biomarcador padrão-ouro (MORILES KE e AZER SA, 2020).

Em indivíduos com lesão muscular grave, o aumento sérico da ALT liberada pela rabdomiólise pode mascarar a atividade da ALT originada no fígado, como consequência do dano hepatocelular (SCHOMAKER S, et al., 2020). Curiosamente, a relação probabilística entre o pico de CPK e a concentração sérica de ALT pode ser uma ferramenta útil em predizer os níveis de ALT a partir da rabdomiólise ou de outras causas (LIM AKH, 2020). Isso sustenta a hipótese de que os elevados níveis de ALT encontrados neste estudo de caso não podem ser justificados exclusivamente pela rabdomiólise, mas, possivelmente, da principal fonte dessa aminotransferase: o hepatócito.

Atualmente, várias abordagens foram propostas para melhorar o diagnóstico do aparecimento de lesão hepática aguda em indivíduos com doenças musculares subjacentes, como a associação de outros biomarcadores hepáticos e exames de imagem, aumentando a interpretação dos resultados da ALT em ensaios clínicos (SCHOMAKER S, et al., 2020; ROS PR, 2018).

A rabdomiólise é um evento clássico do acidente crotálico, porém, quando maciça (evolução incomum), pode induzir à lesão hepática aguda. Esta, por sua vez, é rara e pode surgir de maneira independente ou se agravar mediante rabdomiólise, pois as evidências levantadas na discussão mostram que peçonha crotálica pode ser hepatotóxica. Assim, novos exames complementares com foco na avaliação da integridade hepática devem ser inseridos na condução diagnóstica. Após o reconhecimento da hepatite aguda, a preservação da função hepática deve ser um dos objetivos no transcurso terapêutico, a fim de garantir um melhor prognóstico e redução da taxa de mortalidade.

\section{REFERÊNCIAS}

1. BARRAVIERA B, et al. A retrospective study of 40 victims of Crotalus snake bites: Analysis of the hepatic necrosis observed in one patient. Revista da Sociedade Brasileira de Medicina Tropical, 1989; 22: 5-12.

2. CARVALHO LH, et al. Local and systemic effects caused by Crotalus durissus terrificus, Crotalus durissus collilineatus, and Crotalus durissus cascavella snake venoms in swiss mice. Revista da Sociedade Brasileira de Medicina Tropical, 2019; 52.

3. FARIA G, LIMA AAM. Aspectos Epidemiológicos Dos Acidentes Por Animais Peçonhentos Ocorridos No Município De Cacoal-RO No Período De 2007-2016. Thêma et Scientia, 2019; 9:122-126.

4. FRANÇA RF, et al. Acute hepatotoxicity of Crotalus durissus terrificus (South American rattlesnake) venom in rats. Journal of Venomous Animals and Toxins including Tropical Diseases, 2009; 15: 61-78. 
5. FRARE BT, et al. Clinical, laboratory, and therapeutic aspects of Crotalus durissus (South American rattlesnake) victims: A literature review. BioMed research international, 2019; 2019: 1-7.

6. GUTIERREZ JM, et al. Snakebite envenoming. Nature reviews Disease primers, 2017; 3: 1-21.

7. HEARD H, BARKER J. Recognizing, diagnosing, and treating rhabdomyolysis. Journal of the American Academy of PAs, 2016; 29: 29-32.

8. JO KM, et al. Serum aminotransferase level in Rhabdomyolysis according to concurrent liver disease. The Korean Journal of Gastroenterology, 2019; 74: 205-211.

9. LIM AKH. Abnormal liver function tests associated with severe rhabdomyolysis. World journal of gastroenterology, 2020; 26(10): 1020.

10. LIMA SC, et al. Pharmacological characterization of crotamine effects on mice hind limb paralysis employing both ex vivo and in vivo assays: Insights into the involvement of voltage-gated ion channels in the crotamine action on skeletal muscles. PLoS neglected tropical diseases, 2018; 12: e0006700.

11. MATOS RR, IGNOTTI E. Incidência de acidentes ofídicos por gêneros de serpentes nos biomas brasileiros. Ciência \& Saúde Coletiva, 2020; 25(7): 2837-2846.

12. MENDONÇA-DA-SILVA I, et al. Safety and efficacy of a freeze-dried trivalent antivenom for snakebites in the Brazilian Amazon: An open randomized controlled phase Ilb clinical trial. PLOS Neglected Tropical Diseases, 2017; 11: e0006068.

13. MINISTÉRIO DA SAÚDE. Acidentes ofídicos: Ministério da Saúde alerta para os impactos dos acidentes relacionados às picadas de cobras. Brasil, 2021. Disponível em: https://www.gov.br/saude/ptbr/assuntos/noticias/2021-1/setembro/ministerio-da-saude-alerta-para-os-impactos-dos-acidentesrelacionados-as-picadas-de-cobras. Acesso em: 15 out. 2021.

14. MINISTÉRIO DA SAÚDE. Secretaria de Vigilância em Saúde, Departamento de Vigilância de Doenças e Agravos Não Transmissíveis e Promoção da Saúde. Saúde Brasil 2014: uma análise da situação de saúde e das causas externas. - Brasília - DF, Brasil, 2015. 462 p. Disponível em: link. Acesso em: 19 out. 2021.

15. MORILES KE, AZER SA. Alanine Amino Transferase. Treasure Island (FL): StatPearls Publishing, 2020.

16. OLIVEIRA IS, et al. Global proteomic and functional analysis of Crotalus durissus collilineatus individual venom variation and its impact on envenoming Análise proteômica e funcional global da variação individual do veneno de Crotalus durissus collilineatus e seu impacto no envenenamento. Journal of Proteomics, 2019; 191: 153-165.

17. PASTORE NETO $M$, et al. Fatores associados à variação da creatina fosfoquinase (CPK) em pacientes vítimas de trauma, submetidos à "Onda Vermelha", com evolução à rabdomiólise. Revista do Colégio Brasileiro de Cirurgiões, 2018; 45: e20181604.

18. PUCCA MB, et al. Crotalus Durissus Ruruima: Current Knowledge on Natural History, Medical Importance, and Clinical Toxinology. Front. Immunol, 2021; 12: 659515.

19. ROS PR. Imaging of Diffuse and Inflammatory Liver Disease. Diseases of the Abdomen and Pelvis 20182021, 2018; 1: 237-246.

20. ROSA AC, et al. Insuficiência renal aguda após acidente crotálico: Relato de caso clínico. Brazilian Journal of Health Review, 2021; 4: 20211-20217.

21. SCHOMAKER S, et al. Serum glutamate dehydrogenase activity enables early detection of liver injury in subjects with underlying muscle impairments. Plos one, 2020; 15: e0229753.

22. SOTO-BLANCO B, MELO MM. Acidente Crotálico. Cadernos Técnicos de Saúde da FASEH, 2018; 5: 710.

23. SOUZA LA, et al. Perfil das vítimas de acidente ofídico notificadas em um hospital público de ensino: estudo transversal. Revista da Escola de Enfermagem da USP, 2021; 55: e03721.

24. TORRES PA, et al. Rhabdomyolysis: pathogenesis, diagnosis, and treatment. Ochsner Journal, 2015; 15: 58-69.

25. VAZ VHS, et al. Propriedade intelectual do soro antiofídico: a efetividade a partir da correlação entre os investimentos do governo federal nos principais institutos responsáveis pela produção do soro e realização de pesquisas para o tratamento de acidentes ofídicos no Brasil, com relação ao número de vítimas fatais dos acidentes. Cadernos Saúde Coletiva, 2020; 28: 409-421. 